\title{
Algorithms for Triangular Decomposition of Block Hankel and Toeplitz Matrices with Application to Factoring Positive Matrix Polynomials
}

\author{
By J. Rissanen
}

\begin{abstract}
Algorithms are given for calculating the block triangular factors $A, \hat{A}, B=A^{-1}$ and $\hat{B}=\hat{A}^{-1}$ and the block diagonal factor $D$ in the factorizations $R=A D \hat{A}$ and $B R \hat{B}=D$ of block Hankel and Toeplitz matrices $R$. The algorithms require $O\left(p^{3} n^{2}\right)$ operations when $R$ is an $n \times n$-matrix of $p \times p$-blocks.

As an application, an iterative method is described for factoring $p \times p$-matrix valued positive polynomials $R=\sum_{i=-m}^{m} R_{i} x^{i}, R_{-i}=R_{i}^{\prime}$, as $\bar{A}(x) \bar{A}^{\prime}\left(x^{-1}\right)$, where $\bar{A}(x)$ is outer.
\end{abstract}

1. Introduction. Let $R=\left\{R_{i i}\right\}, i, j=0,1, \cdots, n$, be a matrix with the entries $R_{i j}$ as $p \times p$-matrices of real valued elements. Such a matrix is called a (block) Hankel matrix if $R_{i j}=R_{i+i}$, and a (block) Toeplitz matrix if $R_{i j}=R_{i-i}$.

When all the block $(m+1) \times(m+1)$-sections $\left\{R_{i j}\right\}, i, j=0,1, \cdots, m, m \leqq n$, of either a Hankel matrix $R$ or a Toeplitz matrix $R$ are invertible, we shall construct the two factorizations,

$$
\begin{aligned}
& R=A D \hat{A}, \\
& B R \hat{B}=D,
\end{aligned}
$$

where $A$ is a lower triangular matrix with $p \times p$-identity matrices $I$ on the diagonal, $\hat{A}$ is an upper triangular matrix with matrices $I$ on the diagonal, $D$ is an invertible block diagonal matrix, and $B$ and $\hat{B}$ are inverses of $A$ and $\hat{A}$, respectively. The algorithms for finding the two triangular decompositions require $O\left(p^{3} n^{2}\right)$ arithmetic operations when $R$ is either a Hankel matrix or a Toeplitz matrix.

Special cases of our algorithms have been derived earlier for different purposes. For $p=1$ and $R$ a positive definite Toeplitz matrix, Levinson [1] has derived such an algorithm for solving predictor problems, which, in effect, also finds the factors (1.2). A generalization of the same algorithm for $p>1$ was derived (slightly imprecisely) in [2]. For $p=1$ and $R$, a Hankel matrix, an algorithm for finding the factors in (1.1) was derived in [3] by use of the so-called moments and the Lanczos algorithm.

The algorithm for finding the factorization (1.1) for $R$, a Toeplitz matrix, is believed to be completely new; not even special cases of it seem to have been found before. In the particular case where $R$ is symmetric and positive definite, this algorithm also finds the related Cholesky factorization, $R=\bar{A} \bar{A}^{\prime}[6]$.

Received October 22, 1971, revised April 15, 1972.

AMS (MOS) subject classifications (1970). Primary 65F30; Secondary 65F25, 15 A57.

Key words and phrases. Hankel and Toeplitz matrices, triangular decomposition of matrices.

Copyright (c) 1973, American Mathematical Society 
The algorithm for the Cholesky factors has an important application in a numerical solution of the classical spectral factorization problem. This problem, with applications in prediction theory, representation or identification of random processes, and a number of other areas as well, is to factor a $p \times p$-matrix polynomial $R(x)=$ $\sum_{i=-m}^{m} R_{i} x^{i}, R_{i}=R_{-i}^{\prime}$, positive for each $|x|=1$, as follows:

$$
R(x)=\bar{A}(x) \bar{A}^{\prime}\left(x^{-1}\right),
$$

where the factor $\bar{A}(x)$ has the form $\sum_{i=0}^{m} \bar{A}_{i} x^{i}$ and is "outer" [4]. In the present context, $\bar{A}(x)$ being outer merely means that it has the indicated form and is invertible for $|x| \leqq 1$. Our algorithm requires an order of magnitude fewer arithmetic operations than the earlier ones in [5] and [7].

2. Hankel Matrices. We begin with the factorizations (1.1)-(1.2) when $R$ is a (blockwise) $(n+1) \times(n+1)$-matrix $\left\{R_{i+j}\right\}$ with all the block $m \times m$-sections $m \leqq n$, nonsingular. Consider the following portion of the matrix equation $B R=$ $D \hat{A}={ }^{\triangle} P$ :

$$
\begin{aligned}
& \left(\begin{array}{ccccc}
I & & & \\
B_{10} & I & & \\
\vdots & \ddots & & \\
B_{k-1,0} & \cdots & I & \\
B_{k 0} & \cdots & B_{k, k-1} & I \\
B_{k+1,0} & \cdots & & B_{k+1, k} & I
\end{array}\right)\left(\begin{array}{cccc}
R_{0} & R_{1} & \cdots & R_{k} \\
R_{1} & R_{2} & \cdots & R_{k+1} \\
\vdots & \vdots & & \vdots \\
R_{k-1} & R_{k} & \cdots & R_{2 k-1} \\
R_{k} & R_{k+1} & \cdots & R_{2 k} \\
R_{k+1} & R_{k+2} & \cdots & R_{2 k+1}
\end{array}\right) \\
& =\left(\begin{array}{cccc}
P_{00} & P_{01} & \cdots & P_{0 k} \\
& \ddots & & \vdots \\
0 & \cdots & 0 & P_{k k} \\
0 & & \cdots & 0
\end{array}\right) .
\end{aligned}
$$

Observe the evident but important fact that these subfactorizations, one for each $k, k \leqq n$, form a nested sequence such that a subfactorization for any $k^{\prime}, k^{\prime} \leqq k$, is also a subfactorization of the one corresponding to $k$.

Denoting the $i$ th block-row of $R$ as the array $\left(R_{i}, R_{i+1}, \cdots\right)$, we find that the $(k+1)$ th block-row of $B$ satisfies the equation and, conversely, is determined by it:

$$
\begin{aligned}
B_{k+1,0}\left(R_{0}, \cdots, R_{k}\right)+\cdots+B_{k+1, k}\left(R_{k}, \cdots,\right. & \left.R_{2 k}\right) \\
& \quad+\left(R_{k+1}, \cdots, R_{2 k+1}\right)=0 .
\end{aligned}
$$

By picking the block columns 1 to $k-1$ from $R$, we also have

$$
\begin{aligned}
B_{k, 0}\left(R_{1}, \cdots, R_{k-1}\right)+\cdots+B_{k, k-1}\left(R_{k}, \cdots,\right. & \left.R_{2 k-2}\right) \\
& +\left(R_{k+1}, \cdots, R_{2 k-1}\right)=0 .
\end{aligned}
$$

With the notations,

$$
\begin{aligned}
& E_{0}=B_{k+1,0}, \\
& E_{i}=B_{k+1, i}-B_{k, i-1}, \quad=1, \cdots, k,
\end{aligned}
$$


we deduce from (2.2) and (2.3), by subtraction, the two equations:

$$
E_{0}\left(R_{0}, \cdots, R_{k-2}\right)+\cdots+E_{k}\left(R_{k}, \cdots, R_{2 k-2}\right)=0, \quad k \geqq 2,
$$

and

$$
B_{k+1,0}\left(R_{k-1}, R_{k}\right)+\cdots+B_{k+1, k}\left(R_{2 k-1}, R_{2 k}\right)+\left(R_{2 k}, R_{2 k+1}\right)=0, \quad k \geqq 1 .
$$

Regard Eqs. (2.5) as $p$ homogeneous equations in the $p$-rows of the block-row $\left(E_{0}, \cdots, E_{k}\right)$. As the sections $\left\{R_{i+j}\right\}, i+j \leqq 2 k$, are nonsingular, we conclude by comparing (2.1) and (2.5) that every row of $\left(E_{0}, \cdots, E_{k}\right)$ must lie in the linear manifold spanned by the $2 p$ linearly independent rows in the two block-rows $\left(B_{k-1,0}, \cdots, I, 0\right)$ and $\left(B_{k, 0}, \cdots, I\right)$ of $B$. Hence, two matrices $C_{k}$ and $G_{k-1}$ exist such that

$$
\left(E_{0}, \cdots, E_{k}\right)=C_{k}\left(B_{k, 0}, \cdots, I\right)+G_{k-1}\left(B_{k-1,0}, \cdots, I, 0\right) .
$$

The coefficient matrices $C_{k}$ and $G_{k-1}$ are determined by (2.6) in the following way: First, (2.4) and (2.6) imply

$$
E_{0}\left(R_{k-1}, R_{k}\right)+\cdots+E_{k}\left(R_{2 k-1}, R_{2 k}\right)+\left(P_{k k}, P_{k, k+1}\right)=0 .
$$

Then, with the preceding equality, we derive the equality

$$
C_{k}\left(0, P_{k k}\right)+G_{k-1}\left(P_{k-1, k-1}, P_{k-1, k}\right)+\left(P_{k k}, P_{k, k+1}\right)=0
$$

which leads to

$$
\begin{aligned}
G_{k-1} & =-D_{k} D_{k-1}^{-1}, & & k \geqq 1, \\
C_{k} & =-\left(G_{k-1} P_{k-1, k}+P_{k, k+1}\right) D_{k}^{-1}, & & k \geqq 0,
\end{aligned}
$$

where $D_{k}=P_{k k}$. Define $G_{i}=0$, if $i$ is negative, and $P_{i j}=0$ and $B_{i j}=0$, if $i$ or $j$ are negative. Then, we get the first recursion:

$$
\begin{aligned}
B_{k+1, i} & =B_{k, i-1}+C_{k} B_{k i}+G_{k-1} B_{k-1, i}, \quad k \geqq 0, i=0, \cdots, k+1, \\
B_{00} & =I,
\end{aligned}
$$

which implies $B_{i i}=I$ for $i \geqq 0$; we put $B_{i j}=P_{i i}=0$ for $j>i$. Rather than calculate $P_{k, k+1}$ and $D_{k}$ from (2.1) as sums, it is better to obtain them as by-products from a recursion for the elements $P_{i j}$ as follows.

Equations (2.1) with (2.8) lead to

$$
\begin{aligned}
P_{j k} & =\sum_{i=0}^{j} B_{i i} R_{k+i} \\
& =P_{i-1, k+1}+C_{i-1} P_{i-1, k}+G_{i-2} P_{i-2, k}, \quad k>0, j=1, \cdots, k, \\
P_{0 k} & =R_{k}, \quad k \geqq 0, \\
D_{k} & =P_{k k} .
\end{aligned}
$$

From $P$, the matrix $\hat{A}$ can be recovered by

$$
\hat{A}=D^{-1} P \text {. }
$$

Equations (2.7), (2.9), and (2.10) generalize those in [3]. Equations (2.7) $-(2.10)$ give both $B$ and $\hat{A}$ in $O\left(2 n^{2}\left(p^{3}+p^{2}\right)\right)$ operations. 
By transposing Eqs. (1.1) and (1.2) we have

$$
R^{\prime}=\hat{A}^{\prime} D^{\prime} A^{\prime}, \quad \hat{B}^{\prime} R^{\prime} B^{\prime}=D^{\prime} .
$$

Therefore, in order to get the factors $\widehat{B}^{\prime}$ and $A^{\prime}$, we just replace $R_{i}$ by $R_{i}^{\prime}$ in all the preceding equations.

3. Toeplitz Matrices. Consider again the following portion of the matrix equation $B R=D \hat{A}={ }^{\Delta} P$, where all the block $k \times k$-sections of the Toeplitz matrix $R$ are nonsingular:

$$
\begin{gathered}
{\left[\begin{array}{cccccc}
I & & & \\
B_{10} & I & & \\
\vdots & & & \\
B_{k 0} & \cdots & B_{k, k-1} & I \\
B_{k+1,0} & \cdots & & B_{k+1, k} & I
\end{array}\right]\left(\begin{array}{cccc}
R_{0} & R_{1} & \cdots & R_{k} \\
R_{-1} & R_{0} & R_{1} & R_{k-1} \\
\vdots & & & \vdots \\
R_{-k} & \cdots & & R_{0} \\
R_{-k-1} & \cdots & & R_{-1}
\end{array}\right]} \\
= \\
=\left(\begin{array}{cccc}
P_{00} & P_{01} & \cdots & P_{0 k} \\
& P_{11} & \cdots & P_{1 k} \\
& & \ddots & \vdots \\
0 & \cdots & 0 & P_{k k} \\
0 & & \cdots & 0
\end{array}\right] .
\end{gathered}
$$

We can then deduce the two equations, which, conversely, determine the block-rows of $B$ :

$$
\begin{aligned}
B_{k+1,0}\left(R_{0}, \cdots, R_{k}\right)+B_{k+1,1}\left(R_{-1}, R_{0}, \cdots, R_{k-1}\right) & \\
& +\cdots+\left(R_{-k-1}, \cdots, R_{-1}\right)=0, \\
B_{k 0}\left(R_{0}, \cdots, R_{k-1}\right)+B_{k 1}\left(R_{-1}, R_{0}, \cdots,\right. & \left.R_{k-2}\right) \\
& +\cdots+\left(R_{-k}, \cdots, R_{-1}\right)=0 .
\end{aligned}
$$

With the notations (2.4), we further deduce from (3.2) (by solving the second equation for $\left(R_{-k}, \cdots, R_{-1}\right)$ and substituting the result in the first:

$$
\begin{array}{r}
E_{0}\left(R_{1}, \cdots, R_{k}\right)+E_{1}\left(R_{0}, \cdots, R_{k-1}\right)+\cdots+E_{k}\left(R_{-k+1}, \cdots, R_{0}\right)=0, \\
B_{k+1,0} R_{0}+B_{k+1,1} R_{-1}+\cdots+R_{-k-1}=0 .
\end{array}
$$

Our plan is to express the first block-row $\left(R_{1}, \cdots, R_{k}\right)$ in the form $Y_{1}\left(R_{0}, \cdots, R_{k-1}\right)$ $+\cdots+Y_{k}\left(R_{-k+1}, \cdots, R_{0}\right)$ where the $Y_{i}$ 's do not involve the $B_{k+1, i}$ 's which we are looking for; for, once this is done, we can deduce from (3.3) an equation of the form

$$
X_{1}\left(R_{0}, \cdots, R_{k-1}\right)+\cdots+X_{k}\left(R_{-k+1}, \cdots, R_{0}\right)=0,
$$

which has only the solution $X_{i}=0$ for $i=1, \cdots, n$. This is true since the blockwise $k \times k$-submatrix of $R$ with the indicated rows is nonsingular by assumption. 
A look at the second equation in (3.2) suggests how the stated goal is achieved: define $R^{*}=\left\{R_{i j}^{*}\right\}$ with $R_{i j}^{*}=R_{i-i}$; i.e., replace in (3.1) $R_{i}$ by $R_{-i} . R^{*}$ is seen to be equivalent to $R$, so that the factorization (1.2), $B^{*} R^{*} \hat{B}^{*}=D^{*}$, exists. Then, as in (3.2),

$$
B_{k 0}^{*}\left(R_{0}, \cdots, R_{-k+1}\right)+B_{k 1}^{*}\left(R_{1}, \cdots, R_{-k+2}\right)+\cdots+\left(R_{k}, \cdots, R_{1}\right)=0,
$$

where the $B_{k i}^{*}$ 's play the role of the $Y_{i}$ 's as planned. By solving (3.5) for $\left(R_{k}, \cdots, R_{1}\right)$ and substituting the result in (3.3), we get

$$
\left(E_{1}-E_{0} B_{k, k-1}^{*}\right)\left(R_{0}, \cdots, R_{k-1}\right)+\cdots+\left(E_{k}-E_{0} B_{k 0}^{*}\right)\left(R_{-k+1}, \cdots, R_{0}\right)=0,
$$

which, since (3.4) has only the trivial solution, holds if and only if

$$
E_{i}-E_{0} B_{k, k-i}^{*}=0, \quad i=1, \cdots, k .
$$

The second equation in (3.3) determines $E_{0}$ as follows: First, (3.3) with (2.4) implies

$$
E_{0} R_{0}+\cdots+E_{k} R_{-k}+\sum_{i=0}^{k} B_{k i} R_{-1-i}=0 .
$$

Then, with (3.6), we get

$$
\begin{array}{rlrl}
E_{0} & =C_{k}=-F_{k} D_{k}^{*-1}, & & k \geqq 0, \\
F_{k} & =\sum_{i=0}^{k} B_{k i} R_{-i-1}, & & k \geqq 0, \\
D_{k}^{*} & =\sum_{i=0}^{k} B_{k i}^{*} R_{i-k}, & k \geqq 0 .
\end{array}
$$

By defining $B_{i j}=B_{i j}^{*}=0$ for $i$ or $j$ negative or $i<j$, and $B_{i i}=B_{i i}^{*}=I$ for $i \geqq 0$, we deduce from (2.4) and (3.6):

$$
B_{k+1, i}=B_{k, i-1}+C_{k} B_{k, k-i}^{*}, \quad i=0, \cdots, k, k \geqq 0 .
$$

Similarly, we obtain

$$
B_{k+1, i}^{*}=B_{k, i-1}^{*}+C_{k}^{*} B_{k, k-i}, \quad i=0, \cdots, k, k \geqq 0,
$$

where

$$
\begin{aligned}
C_{k}^{*} & =-F_{k}^{*} D_{k}^{-1}, \quad k \geqq 0, \\
F_{k}^{*} & =\sum_{i=0}^{k} B_{k i}^{*} R_{i+1}, \quad k \geqq 0, \\
D_{k} & =\sum_{i=0}^{k} B_{k i} R_{k-i} .
\end{aligned}
$$

Further, $D_{k}$ and $D_{k}^{*}$ satisfy by (3.7) and (3.9) the recursions

$$
\begin{aligned}
D_{k+1} & =D_{k}+C_{k} F_{k}^{*}, \quad k \geqq 0, \\
D_{0} & =R_{0}, \\
D_{k+1}^{*} & =D_{k}^{*}+C_{k}^{*} F_{k}, \quad k \geqq 0, \\
D_{0}^{*} & =R_{0} .
\end{aligned}
$$


Equations (3.8)-(3.12) describe an algorithm for finding the factor $B$ in (1.2). Again, $\hat{B}^{\prime}$ can be obtained from the same formulas by replacing $R_{i}$ by $R_{-i}^{\prime}$. Hence, in particular, if $R$ is a symmetric Toeplitz matrix and $R_{i}=R_{-i}^{\prime}$, then $\hat{B}^{\prime}=B$. But even another simplification results: as was shown by J. P. Burg (unpublished),

$$
F_{k}^{*}=F_{k}^{\prime} \quad\left(\text { if } R_{-i}=R_{i}^{\prime}\right) .
$$

To see this, observe that with (3.2) and (3.5) we have

$$
F_{k}^{*}=\left(I, B_{k, k-1}^{*}, \cdots, B_{k 0}^{*}, 0\right)\left(\begin{array}{cccc}
R_{0} & R_{1} & \cdots & R_{k+1} \\
R_{1}^{\prime} & R_{0} & \cdots & R_{k} \\
\vdots & & & \vdots \\
& & & \\
R_{k+1}^{\prime} & & \cdots & R_{0}
\end{array}\right)\left(\begin{array}{c}
0 \\
B_{k 0}^{\prime} \\
\vdots \\
B_{k, k-1}^{\prime} \\
I
\end{array}\right)=F_{k}^{\prime} .
$$

We point out that it is possible to derive a recurrence equation also for $F_{k}$ and $F_{k}^{*}$, as we shall show shortly. However, these recurrence relations save calculations only when both factorizations (1.1) and (1.2) are determined.

We shall now turn to the problem of finding the factors in (1.1). The situation turns out to be different from the case with Hankel matrices in that the algorithms for determining the factorizations (1.1) and (1.2), respectively, are independent from each other. Beginning with (3.1), we have

$$
\begin{aligned}
P_{i, k+1} & =\sum_{i=0}^{i} B_{i j} R_{k+1-i}, & & 1 \leqq i \leqq k+1, \\
P_{0 k} & =R_{k}, & & k \geqq 0 .
\end{aligned}
$$

An application of (3.8) to this expression leads to

$$
\begin{aligned}
P_{i, k+1}= & C_{i-1} R_{k+1}+R_{k+1-i} \\
& +\sum_{j=1}^{i-1} B_{i-1, i-1} R_{k+1-i}+C_{i-1} \sum_{j=1}^{i-1} B_{i-1, i-1-i}^{*} R_{k+1-i}, \quad k \geqq 0 .
\end{aligned}
$$

By adding $R_{k+1-i}$ to the first sum and writing

$$
Q_{i k}=\sum_{j=0}^{i} B_{i j}^{*} R_{k-i+j+1}, \quad i=0, \cdots, k, k \geqq 0,
$$

for the second sum joined with $R_{k+1}$ (with $i-1$ replaced by $i$ ), we obtain

$$
\begin{aligned}
P_{i+1, k+1} & =P_{i k}+C_{i} Q_{i k}, & & k \geqq 0, i=0, \cdots, k, \\
P_{0 k} & =R_{k}, & k \geqq 0 . &
\end{aligned}
$$

An application of (3.9) to (3.16) gives in turn the recursion for the $Q_{i k}$ 's:

$$
\begin{aligned}
Q_{i+1, k} & =Q_{i k}+C_{i}^{*} P_{i k}, & & k \geqq 1, i=0, \cdots, k-1, \\
Q_{0 k} & =R_{k+1}, & k & \geqq 0 .
\end{aligned}
$$

Now observe from (3.16) and (3.15): 


$$
\begin{aligned}
Q_{k k}=F_{k}^{*}, & k \geqq 0, \\
P_{k k}=D_{k}, & k \geqq 0 .
\end{aligned}
$$

In order to also get $F_{k}$ recursively, replace, in (3.15)-(3.16), $R_{i}$ by $R_{-i}$ and $B_{i j}$ by $B_{i j}^{*}$. Then,

$$
\begin{aligned}
P_{i+1, k+1}^{*} & =P_{i k}^{*}+C_{i}^{*} Q_{i k}^{*}, & & k \geqq 0, i=0, \cdots, k, \\
P_{0 k}^{*} & =R_{-k}, & k \geqq 0, &
\end{aligned}
$$

and

$$
\begin{aligned}
Q_{i+1, k}^{*} & =Q_{i k}^{*}+C_{i} P_{i k}^{*}, & & k \geqq 1, i=0, \cdots, k-1, \\
Q_{0 k}^{*} & =R_{-k-1}, & & k \geqq 0 .
\end{aligned}
$$

Further,

$$
\begin{aligned}
Q_{k k}^{*}=F_{k}, & k \geqq 0, \\
P_{k k}^{*}=D_{k}^{*}, & k \geqq 0 .
\end{aligned}
$$

Finally, we now can express $C_{i}$ and $C_{i}^{*}$ as follows:

$$
\begin{array}{rlrl}
C_{k} & =-Q_{k k}^{*} P_{k k}^{*-1}, & k \geqq 0, \\
C_{k}^{*}=-Q_{k k} P_{k k}^{-1}, & k \geqq 0 .
\end{array}
$$

With

$$
\hat{A}=D^{-1} P,
$$

Eqs. (3.17)-(3.23) describe an algorithm for the factor $\hat{A}$ in (1.1). By replacing $R_{i}$ by $R_{-i}^{\prime}$, the same algorithm also finds the factor $A^{\prime}$. In particular, when $R$ is a symmetric Toeplitz matrix, $\hat{A}^{\prime}=A$, and (3.14) gives

$$
Q_{k k}^{*}=Q_{k k}^{\prime},\left(\text { if } R_{-i}=R_{i}^{\prime}\right) \text {. }
$$

These recurrence relations require $O\left(4 n^{2}\left(p^{3}+p^{2}\right)\right)$ arithmetic operations to determine the factorization (1.1), and only $O\left(5 n^{2}\left(p^{3}+p^{2}\right)\right)$ to find both (1.1) and (1.2) in case $p>1$. In case $p=1$, they require only $O\left(2 n^{2}\right)$ for $(1.1)$ and $O\left(3 n^{2}\right)$ for both (1.1) and (1.2).

In case $R$ is a symmetric positive definite matrix, the factorization

$$
R=\bar{A} \bar{A}^{\prime}
$$

is called a Cholesky factorization [6]. Such a factorization is obtained from (1.1) by first factoring $D$ as

$$
D=G G^{\prime} \text {, }
$$

where $G$ may be taken to be lower triangular with positive elements on the diagonal, and by then putting

$$
\bar{A}=A G .
$$

Therefore, with this addition, our algorithm calculates even the Cholesky factorizations of symmetric positive definite Teoplitz matrices. 
As was shown in [5] for $p=1$ and in [7] for $p \geqq 1$, the Cholesky factorization has a close connection with the classical spectral factorization problem: Let $R=\left\{R_{i j}\right\}$, $i, j=0,1, \cdots, R_{i i}=R_{i-i}$ and $R_{i}=0$ for $i>m$, be the infinite symmetric positive definite block Toeplitz matrix obtained from the ceofficients of a $p \times p$-matrix polynomial $R(x)=\sum_{i=-m}^{m} R_{i} x^{i}, R_{i}=R_{-i}^{\prime}$, which is positive definite for $|x|=1$. Then, as proved in [7], the rows of the Cholesky factor $\bar{A}$ of $R$ converge:

$$
\left(\bar{A}_{n, n-m}, \cdots, \bar{A}_{n n}\right) \rightarrow\left(\bar{A}_{m}, \cdots, \bar{A}_{0}\right) \text { as } n \rightarrow \infty \text {. }
$$

The limits $\bar{A}_{i}$ define the outer factor $\bar{A}(x)=\sum_{i=0}^{n} \bar{A}_{i} x^{i}$ of the spectral factorization (1.3) of $R(x)$.

In this way, the algorithm (3.17)-(3.23) with (3.27)-(3.28), where now $P_{i k}=0$ for $k-i>m$ and $Q_{i k}=0$ for $k-i \geqq m$, provides a new method for calculating the spectral factors with any desired accuracy. These formulas require $O\left(p^{3} m\right)$ operations for each block-row of $\bar{A}$ as compared with $O\left(p^{3} \mathrm{~m}^{2}\right)$ with the earlier algorithms in [5] and [7] which basically are Gauss-type elimination schemes. Finally, in this case, our algorithm (just as that in [5] and [7]) is also numerically stable as can be shown by standard stability arguments.

IBM Corporation

Monterey \& Cottle Roads

San Jose, California 95114

1. N. LEVINSON, "The Wiener RMS error criterion in filter design and prediction," Appendix B of N. Wiener's book Extrapolation, Interpolation, and Smoothing Stationary Time Series with Engineering Applications, Wiley, New York, 1949, pp. 129-148.

2. R. A. Wiggins \& E. A. Robinson, "Recursive solution to the multichannel filtering problem," J. Geophys. Res., v. 70, 1965, pp. 1885-1891. MR 32 \#589.

3. J. L. PhIllips, "The triangular decomposition of Hankel matrices," Math Comp., v. 25,1971 , pp. 599-602.

4. A. DEvinatz, "The factorization of operator valued functions," Ann. of Math., (2), v. 73, 1961, pp. 458-495. MR 23 \#A3997.

5. F. L. BAUER, "Ein direktes Iterationsverfahren zur Hurwitz-Zerlegung eines Polynoms," Arch. Elek. Ubertr., v. 9, 1955, pp. 285-290. MR 17, 900.

6. J. H. WILKINSON \& C. REINSCH, Handbook for Automatic Computation. Vol. II. Linear Algebra, Springer-Verlag, New York, 1971.

7. J. Rissanen \& L. Barbosa, "Properties of infinite covariance matrices and stability of optimum predictors," Information Sci., v. 1, 1969, pp. 221-236. MR 39 \#5032. 